\title{
Effect of Crustal Structure on Rayleigh Waves Generated by Atmospheric Explosions
}

\author{
David G. Harkrider \\ Department of Geological Sciences, Brown University \\ Providence, Rhode Island 02912
}

Edward A. Flinn

Geotech, Alexandria, Virginia 22313

\begin{abstract}
Theoretical seismograms are calculated at a teleseismic distance from atmospheric sources over oceanic and continental earth models. Vertical surface displacements of the fundamental and first higher-mode Rayleigh waves are obtained for each of the models. Source altitudes range from 0.3 to $4.88 \mathrm{~km}$ for a 1-kiloton nuclear explosion in a stratified thermal atmosphere. At 20-sec period, an explosion over the oceanic model exhibits amplitudes an order of magnitude greater than the equivalent amplitudes from an explosion of the same burst height and yield over any of the three continental structures. If the differences in anelastic attenuation over the paths are included, this effect will be reversed at large enough distances.
\end{abstract}

The amplitudes of Rayleigh waves generated by nuclear explosions have frequently been used to estimate their yields [e.g., Bäth, 1962; Toksöz and BenMenahem, 1964; Crampin, 1966]. The theoretical basis for these estimates was a point source in a homogeneous fluid overlying a homogeneous solid half-space. As long as the explosions are at low altitudes and relative estimates for the yields at the same source and receiver locations are desired, this model should provide meaningful results. This is especially true for the technique of Toksöz and Ben-Menahem [1964], even when detonation occurs over water and the receiver is on land, since the effect of the propagation path is eliminated by taking spectral ratios.

In addition, Nickel and Whitaker [1968] developed a technique using the calculated pressure history for a high-altitude source in a realistic atmosphere as a distributed source on the free surface of a solid half-space. The effect of Rayleigh-wave dispersion was modeled by assuming for each period a homogeneous medium with elastic properties that correspond to the observed Rayleigh-wave phase velocity at that period. This model restricted their results to continental source and receiver locations.

Since nuclear explosions have occurred over the ocean as well as the land, there is a need for determining the effect of the region below the source in exciting and propagating Rayleigh waves. In this paper we present the theoretical amplitudes for Rayleigh waves generated by a 1-kT nuclear explosion at altitudes in a stratified thermal atmosphere from 0.3 to $4.88 \mathrm{~km}$ above oceanic and land models. 
In order to make realistic comparisons for land-based stations, the effect of propagation across continental margins must be evaluated. This will be estimated using the observations and theoretical calculations of $M c G a r r$ [1969].

\section{THEORETICAL NUMERICAL METHODS}

\section{Theoretical Formulation}

As in earlier work [Harkrider, 1964a], the thermally stratified atmosphere is modeled by gravitating isothermal layers. The earth models are represented by a multilayered solid elastic half-space. The atmosphere is coupled to the solid earth requiring continuity of pressure and vertical displacement across their interface. For oceanic models, the ocean is included as a layered gravitating liquid in the lower part of the atmospheric wave guide [Harkrider and Press, 1967].

At the periods of interest, 5-60 sec, the vertical dependence of Rayleigh waves in the plane layered medium is a valid approximation to the radial dependence of pressure and particle velocity in the spherical earth. The Rayleigh wave solutions for a source and receiver in the plane layered structure are corrected for sphericity by using the colatitude dependence which, near the source, approaches the flat-earth radial distance dependence [Harkrider, 1964a].

The effect of attenuation along the propagation path due to anelasticity in the solid earth is contained in a spectral decay factor.

The atmosphere is terminated at altitude by a free surface. The rationale behind this assumption will be discussed later.

The assumed source is the Fourier transform in time of the azimuthally symmetric Green's function for a gravitating isothermal space. (For atmospheric symbols used without definition here, refer to Harkrider [1964a]; for solid-earth symbols used without definition, refer to Harkrider [1964b]. The source is in layer $s$ at an altitude $z=D$ and at $r=0$ (Figure 1). The Green's function or point source is normalized so that at a distance $a_{8}$ to the side of the source, i.e., $(r, z)=\left(a_{s}, D\right)$, the spectral source pressure is given by the transformed empirical pressure-time history [Glasstone, 1962].

The resulting spectral source overpressure is given by:

$$
\left\langle p_{s_{0}}\right\rangle=i p_{a} e^{\kappa_{s} a_{a}} e^{-i \omega t_{a} \cdot} \frac{a_{s} \omega}{\left(i_{\omega}+b_{s}\right)^{2}} e^{-\lambda_{s}(z-D)} \cdot \frac{\exp \left\{-\kappa_{s}\left[r^{2}+h_{s}(z-D)^{2}\right]^{1 / 2}\right\}}{\left[r^{2}+h_{s}(z-D)^{2}\right]^{1 / 2}}
$$

At $r=a_{s}$ and $z=D$, (1) reduces to

where

$$
\left\langle p_{s_{0}}\right\rangle=p_{a_{s}} e^{-\imath \omega l_{a s}} \frac{i \omega}{\left(i \omega+1 / T_{+a s}\right)^{2}}=\int_{-\infty}^{\infty}\left[p_{s o}\left(a_{o}, D ; t\right)\right] e^{-\imath \omega t} d t
$$

$$
\begin{gathered}
{\left[p_{s,}\left(a_{a}, D ; t\right)\right]= \begin{cases}p_{a s}\left(1-\tau / T_{+a s}\right) e^{-\tau / T_{+a s}} & \text { for } \tau \geq 0 \\
0 & \text { for } \tau<0\end{cases} } \\
b_{s}=\left(T_{+a s}\right)^{-1} \\
\tau=t-t_{a s}
\end{gathered}
$$




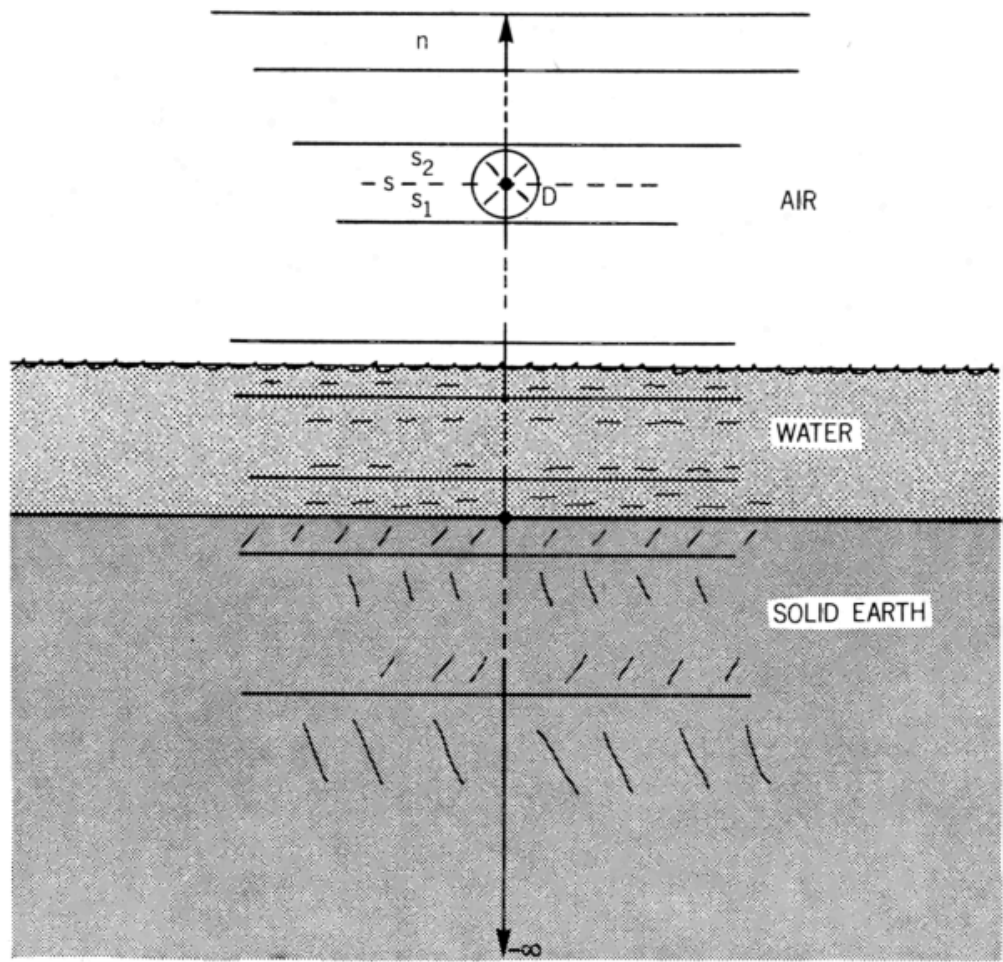

Fig. 1. Geometry of source and mediums.

The peak overpressure $p_{a s}$ is chosen at a distance $a_{s}$ for a 1-kT nuclear explosion, well within the linear zone. For different yields and altitudes, the distance $a_{s}$, the positive phase duration time $T_{+a s}$ and the arrival time $t_{a s}$ are scaled using the scaling rules described by Glasstone [1962]. The peak overpressure is also scaled by the ratio of ambient pressure at the different source altitudes. Scaling the source at the burst height rather than above or below it eliminates the necessity of correcting for the differences in ambient-atmosphere properties at the burst and scale point. This correction can be quite large when scaling below the explosion for large yields and high altitudes.

The condition that $\operatorname{Re}\left(\kappa_{8}\right)>0$ and $\operatorname{Re}\left(h_{8}^{1 / 2}\right)>0$ enables us to use the integral relation [Erdelyi et al., 1954]

$\frac{\exp \left\{-\kappa_{\varepsilon}\left[r^{2}+h_{s}(z-D)^{2}\right]^{1 / 2}\right\}}{\left[r^{2}+h_{s}(z-D)^{2}\right]^{1 / 2}}=h_{s}^{1 / 2} \int_{0}^{\infty} \frac{\exp \left(-i k r_{\alpha s}|z-D|\right)}{i k r_{\alpha s}} J_{0}(k r) k d k$

where

$$
\begin{aligned}
\left(k r_{\alpha_{s}}\right)^{2} & =\omega^{2} / \alpha_{s}{ }^{2}-h_{s} k^{2}-\lambda_{s}{ }^{2} \\
\kappa_{s}{ }^{2} & =\frac{\left(\alpha_{s}{ }^{2} \lambda_{s}{ }^{2}-\omega^{2}\right)}{h_{s} \alpha_{s}^{2}} \\
h_{s} & =\left(1-\sigma_{2}{ }^{2} / \omega^{2}\right) \\
\lambda_{s} & =\gamma g_{s} / 2 \alpha_{s}{ }^{2}
\end{aligned}
$$


and $\sigma_{2}$ is the isothermal Brunt frequency. Rewriting (1) using (4), we have

$$
\left\langle p_{s_{0}}\right\rangle=\int_{0}^{\infty} S_{0}(\omega, k) \exp \left[-\lambda_{s}(z-D)\right] \exp \left(i k r_{a s}|z-D|\right) J_{0}(k r) d k
$$

where $S_{0}(\omega, k)$ is defined by comparison of (6) with (1) and (4). This form of the source is convenient because $r$-dependent and $z$-dependent factors in the integrand are separable solutions of the azimuthally symmetric homogeneous differential equations governing the overpressure in the isothermal layers of the atmosphere:

$$
\frac{1}{r} \frac{\partial}{\partial r}\left(r \frac{\partial p}{\partial r}\right)+\frac{1}{h}\left(\frac{\partial^{2} p}{\partial z^{2}}+2 \lambda \frac{\partial p}{\partial z}+\frac{\omega^{2}}{\alpha^{2}} p\right)=0
$$

Assuming a radial dependence $J_{0}(k r)$, the $z$-dependent part of the homogeneous overpressure $p_{m}(z)$ in layer $m$ is, from (7), a solution of the equation

$$
\frac{\partial^{2} p_{m}}{\partial z^{2}}+2 \lambda_{m} \frac{\partial p_{m}}{\partial z}+\left(\frac{\omega^{2}}{\alpha_{m}^{2}}-h_{m} k^{2}\right) p_{m}=0
$$

The $z$-dependent part of the vertical particle velocity is related to $p_{m}(z)$ by

$$
v_{m}(z)=\frac{i}{\omega \rho_{m}{ }^{0}(z) h_{m}}\left(\frac{\partial p_{m}}{\partial z}+\frac{g_{m}}{\alpha_{m}{ }^{2}} p_{m}\right)
$$

The linearized change in total pressure $p_{P_{m}}{ }^{(2)}$ at an interface which is displaced a vertical distance $-i v_{m} / \omega$ from its equilibrium position is given by

$$
p_{P m}(z)=p_{m}-\frac{g_{m}}{\omega^{2} h_{m}}\left(\frac{\partial p_{m}}{\partial z}+\frac{g_{m}}{\alpha_{m}{ }^{2}} p_{m}\right)
$$

Thus at layer interfaces not containing sources, the boundary condition of continuity of vertical particle velocity and total pressure requires

$$
\left[\begin{array}{c}
v_{m}\left(z_{m-1}\right) \\
p_{P_{m}}\left(z_{m-1}\right)
\end{array}\right]=\left[\begin{array}{c}
v_{m-1}\left(z_{m-1}\right) \\
p_{P m-1}\left(z_{m-1}\right)
\end{array}\right]
$$

For a layer $m$ not containing a source, the pressure and velocity at the top of a layer are related to the pressure and velocity at the bottom of the layer by the matrix relation

$$
\left[\begin{array}{c}
v_{m}\left(z_{m}\right) \\
p_{P_{m}}\left(z_{m}\right)
\end{array}\right]=\mathfrak{a}_{m}\left[\begin{array}{c}
v_{m}\left(z_{m-1}\right) \\
p_{P m}\left(z_{m-1}\right)
\end{array}\right]
$$

where the elements of the layer matrix $a_{m}$ are given by

$$
\begin{aligned}
& a_{11}=e^{\lambda d}\left[\cos P-\left(\lambda-g / c^{2}\right) d \sin P / P\right] \\
& a_{12}=i \frac{d}{\omega \rho^{0}}\left(k^{2}-\frac{\omega^{2}}{\alpha^{2}}\right) \frac{\sin P}{P} \\
& a_{21}=i\left(\rho^{0} / \omega^{3}\right) d \delta \sin P / P \\
& a_{22}=e^{-\lambda d}\left[\cos P+\left(\lambda-g / c^{2}\right) d \sin P / P\right]
\end{aligned}
$$


where

$$
P=k r_{a} d \quad \delta=g^{2} k^{2}-\omega^{4} \quad c=\omega / k
$$

$d$ is the layer thickness, and the layer constants are chosen to correspond to the values at the layer midpoints in the stratified atmosphere model. The subscript $m$ has been suppressed for ease in notation.

For the gravitating ocean layers, the linearized equation for excess pressure $p(z)$ is

$$
\frac{d^{2} p}{d z^{2}}+\frac{g}{\alpha^{2}} \frac{d p}{d z}+\left(\frac{\omega^{2}}{\alpha^{2}}-h k^{2}\right) p=0
$$

where $\alpha$ is the sound velocity in the layer. The relation corresponding to (9) is

$$
v=\frac{i}{\omega \rho^{0} h}\left(\frac{\partial p}{\partial z}+\frac{g}{\alpha^{2}} p\right)
$$

Comparing (15) and (16) with (8) and (9), respectively, we see that the ocean layers can be included in the atmospheric wave guide if we simply let the specific heat ratio $\gamma$ approach unity in all relations except in the definition of the isothermal Brunt frequency.

The general solutions in layer $m$ can be expressed in integral form as

$$
\begin{aligned}
& \left\langle p_{m}\right\rangle=\int_{0}^{\infty} p_{m}(z) J_{0}(k r) d k \\
& \left\langle v_{m}\right\rangle=\int_{0}^{\infty} v_{m}(z) J_{0}(k r) d k
\end{aligned}
$$

and in the source layer $s$ as

$$
\begin{aligned}
& \left\langle p_{s}\right\rangle=\left\langle p_{s 0}\right\rangle+\int_{0}^{\infty} p_{s}(z) J_{0}(k r) d k \\
& \left\langle v_{s}\right\rangle=\left\langle v_{s 0}\right\rangle+\int_{0}^{\infty} v_{s}(z) J_{0}(k r) d k
\end{aligned}
$$

where the integrand of $\left(v_{s 0}\right)$ is obtained by applying (9) to (6), and $p_{s}(z)$ and $v_{s}(z)$ are solutions to the homogeneous equations. Evaluating (18) just above and just below the source altitude $z=D$, we find that the $z$-dependent integrands are related by the vector equation

$$
\left[\begin{array}{c}
v_{\mathrm{a}_{+}}(D) \\
p_{P_{+}+}(D)
\end{array}\right]=\left[\begin{array}{c}
v_{\mathrm{a}-}(D) \\
p_{P_{\mathrm{s}}}(D)
\end{array}\right]+\left[\begin{array}{c}
\Delta v_{\mathrm{s}} \\
\Delta p_{P_{\mathrm{a}}}
\end{array}\right]
$$

where

$$
\begin{aligned}
\Delta v_{s} & =\frac{2 k r_{\alpha s} S_{0}}{\omega h_{s} \rho_{s}{ }^{\circ}(D)} \\
\Delta p_{P_{s}} & =\frac{i g_{s} \rho_{s}^{0}(D)}{\omega} \Delta v_{s}
\end{aligned}
$$


and the subscripts $s_{+}$and $s_{-}$indicate the region in the source layer above and below the source, respectively. The partial derivatives with respect to $z$ used in obtaining (19) were evaluated at $D$ by letting $z \rightarrow D$ from their respective regions.

In the region above the source, $v_{s+}(z)$ and $p_{P_{s+}}(z)$ at the top of the $s$ layer and at $z=D$ are related by

$$
\left[\begin{array}{c}
v_{s+}\left(z_{s}\right) \\
p_{P_{s}+}\left(z_{s}\right)
\end{array}\right]=a_{s+}\left[\begin{array}{c}
v_{s+}(D) \\
p_{P_{s}}(D)
\end{array}\right]
$$

where $a_{s+}$ is given by (13) with the layer thickness $d$ equal to $z_{\mathrm{a}}-D$. A similar relation holds for the region below the source. The product of the layer matrices above and below the source can be shown to yield

$$
a_{8+} a_{s-}=a_{8}
$$

where $Q_{s}$ is the $s$ layer matrix for $d=z_{s}-z_{s-1}$.

The continuity relations (11) across layer interfaces require that

$$
\begin{gathered}
{\left[\begin{array}{c}
v_{n}\left(z_{n}\right) \\
p_{P_{n}}\left(z_{n}\right)
\end{array}\right]=A_{s+}\left[\begin{array}{c}
v_{s+}(D) \\
p_{P_{s+}}(D)
\end{array}\right]} \\
{\left[\begin{array}{c}
v_{s-}(D) \\
p_{P_{s+}}(D)
\end{array}\right]=A_{s-}\left[\begin{array}{c}
v_{0} \\
p_{P_{0}}
\end{array}\right]}
\end{gathered}
$$

where

$$
\begin{aligned}
A_{s+} & =\mathfrak{Q}_{n} \cdots \mathfrak{Q}_{s+} \\
A_{s-} & =\mathfrak{Q}_{s-} \cdots \mathfrak{Q}_{1}
\end{aligned}
$$

The subscript $n$ denotes the uppermost layer and $v_{0}$ and $p_{P_{0}}$ are the vertical particle velocity and partial pressure at the solid earth-atmosphere interface.

Defining $X$ and $Y$ by

$$
\left[\begin{array}{l}
X \\
Y
\end{array}\right] \equiv A_{s-}^{-1}\left[\begin{array}{c}
v_{a_{+}}(D) \\
p_{P_{\mathrm{a}}}(D)
\end{array}\right]
$$

and substituting (24) and (26) into (19), we obtain

$$
\left[\begin{array}{l}
X \\
Y
\end{array}\right]=\left[\begin{array}{c}
v_{0} \\
p_{P_{0}}
\end{array}\right]+A_{s-}{ }^{-1}\left[\begin{array}{c}
\Delta v_{\bullet} \\
\Delta p_{P_{\mathrm{a}}}
\end{array}\right]
$$

The free-surface boundary condition assumed at the top of the atmosphere is

$$
\left[\begin{array}{c}
v_{n}\left(z_{n}\right) \\
p_{P_{n}}\left(z_{n}\right)
\end{array}\right]=\left[\begin{array}{c}
v_{n} \\
\mathbf{0}
\end{array}\right]
$$

Combining (28) with (23) and (26), we have

$$
\left[\begin{array}{l}
v_{n} \\
0
\end{array}\right]=A_{s+} A_{s-}\left[\begin{array}{l}
X \\
Y
\end{array}\right] \equiv A\left[\begin{array}{l}
X \\
Y
\end{array}\right]
$$


where by (25) and (22)

$$
A=a_{n} \cdots a_{s} \cdots a_{1}
$$

From (29)

$$
X=-\left(A_{22} / A_{21}\right) Y
$$

which, in combination with (27), yields

$$
\begin{gathered}
v_{0}=-\frac{\left[A_{22} B+A_{21} E\right]}{\left[A_{21}+\frac{p_{P_{0}}}{v_{0}} A_{22}\right]} \\
B \equiv-\Delta v_{s}\left[\left(A_{s-}\right)_{21}-\frac{i g_{s} \rho_{s}^{0}}{\omega}\left(A_{s-}\right)_{11}\right]
\end{gathered}
$$

where

$$
\begin{gathered}
E \equiv \Delta v_{s}\left[\left(A_{s-}\right)_{22}-\frac{i g_{s} \rho_{s}^{0}}{\omega}\left(A_{s-}\right)_{12}\right] \\
\frac{\Delta p_{P_{s}}}{\Delta v_{s}}=\frac{i g_{s} \rho_{s}^{0}}{\omega} \\
A_{s-}{ }^{-1}=\left[\begin{array}{rr}
\left(A_{s-}\right)_{22} & -\left(A_{s-}\right)_{12} \\
-\left(A_{s-}\right)_{21} & \left(A_{s-}\right)_{11}
\end{array}\right]
\end{gathered}
$$

In terms of the normal stress $\sigma_{0}$ and the vertical positive downward displacement $w_{0}$ at the earth-atmosphere interface,

$$
\frac{p_{P_{0}}}{v_{0}}=\frac{1}{c}\left[\frac{\sigma_{0}}{\dot{w}_{0} / c}\right] \equiv \frac{1}{c}\lfloor T]
$$

where for the solid layers teminated at depth by a half-space [Harkrider, 1964b; Harkrider, 1969]

$$
[T]=-\frac{[N K-L M]}{[G N-L H]}=-\frac{\mathfrak{R}_{11}}{\mathfrak{R}_{12}}
$$

The quantities $N, K, L, M, G$, and $H$ are linear combinations of the elements of the product matrix for solid layers [Harkrider, 1964b], and $\mathcal{R}_{11}$ and $\mathcal{R}_{12}$ are elements of their compound product matrix [Thrower, 1965; Dunkin, 1965; Gilbert and Backus, 1966; and Harkrider, 1969].

Rearranging (32) using (35) and (36), we obtain

$$
\dot{w}_{0}=-c \frac{\left\{A_{22} B+A_{21} E\right\}[G N-L H]}{A_{22} F_{e}}
$$

where

$$
\begin{gathered}
F_{s} \equiv[N K-L M]-c\left(A_{21} / A_{22}\right)[G N-L H] \\
\dot{w}_{0}=-v_{0}
\end{gathered}
$$


Thus

$$
\left\langle w_{0}\right\rangle=\int_{0}^{\infty} 2 \hat{S}_{0}(\omega, k) \frac{N_{\bullet}}{F_{\bullet}} J_{0}(k r) d k
$$

where

$$
\begin{gathered}
\hat{S}_{0} \equiv i \frac{a_{s} p_{a s}}{\rho_{s}^{0}(D)} \frac{e^{\alpha_{s} a_{s}}}{h_{s}^{1 / 2}\left(b_{s}+i \omega\right)^{2}} e^{-i \omega c_{a s}} \\
\left.+\left(A_{21} / A_{22}\right)\left[\left(A_{s-}\right)_{22}-i \frac{g_{s} \rho_{s}^{0}}{\omega}\left(A_{s-}\right)_{12}\right]\right\} \cdot[G N-L H]
\end{gathered}
$$

Evaluating the residue contribution of the integral (39) at the poles where $F_{e}\left(\omega, k_{j}\right)=0$, we obtain the spectral component of the surface-wave displacement:

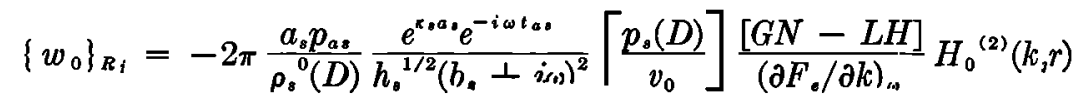

In obtaining (42) we have used the relation $F_{e}\left(\omega, k_{j}\right)=0$, or by $(38),(36)$, and (35),

$$
[T]=-\frac{[N K-L M]}{[G N-L H]}=-c \frac{A_{21}}{A_{22}}
$$

or

$$
\frac{p_{P_{0}}}{v_{0}}=-\frac{p_{P 0}}{\dot{w}_{0}}=-\frac{A_{21}}{A_{22}}
$$

Equations (9) and (10) yield

$$
p_{P m}=p_{m}+i \frac{\rho_{m}^{0} g_{m}}{\omega} v_{m}
$$

which, in combination with equations (44) and (24), reduces $N_{e}$ at the $k_{j}$ root to

$$
N_{e,}=-\frac{p_{s}(D)}{v_{0}}[G N-L H]
$$

which was used in (42).

Thus the time-domain representation of the spectral surface-wave displacement in the far field is

$$
\begin{aligned}
{\left[w_{0}\right]_{h_{s}}=-\left(\frac{2}{\pi}\right)^{1 / 2} \int_{-\infty}^{\infty} \frac{a_{s} p_{a s}}{\rho_{s}^{0}(D)}\left[\frac{p_{s}(D)}{v_{0}}\right] \frac{[G N-L H]}{\left(\partial F_{s} / \partial k\right)_{\omega}} \frac{e^{\kappa_{s} a_{s}}}{h_{s}^{1 / 2}\left(b_{s}+i \omega\right)^{2}} } \\
\cdot\left(\frac{r}{a_{e} \sin \theta}\right)^{1 / 2} \frac{\exp \left[i\left(\omega t-k r+\pi / 4-\omega t_{a s}\right)\right]}{(k r)^{1 / 2}} d \omega
\end{aligned}
$$

where we have used the asymptotic expansion for large arguments of the Hankel 
function and an approximation for curvature; $a_{\bullet}$ denotes the radius of the earth, and $\theta$ is the colatitude.

An alternate form in terms of the solid earth compound product matrix $R$ can be obtained using the following relations [Harkrider, 1970]:

$$
\begin{aligned}
N K-L M=-\mathfrak{R}_{11} \quad G N-L H & =-\mathfrak{R}_{12} \quad R N-S L=-\mathfrak{R}_{13} \\
G M-H K & =-\mathbb{R}_{14}
\end{aligned}
$$

\section{Numerical Procedure}

The vertical displacements at the atmosphere-solid earth interface for land and oceanic models given by (47) were calculated by means of three Fortran programs.

The first program, written for the CDC 1604, calculates the roots $k_{j}$ of $F_{e}$ $=0$ for an input period or frequency. The program is a patching together of the multilayered solid half-space program described by Harkrider [1970] and the multilayered atmosphere program described by Press and Harkrider [1962]. The solid-layer part of the program uses the compound layer matrix formulation.

Once a root is found to the required accuracy, the group velocity, $\left(\partial F_{\sigma} / \partial k\right)_{\omega} \omega$, $[G N-L H]$, and $\left[p_{P_{0}} / v_{0}\right]$ are calculated. The group velocity $U$ and $\left(\partial F_{o} / \partial k\right)_{\omega}$ are formed by computing the analytic derivatives with respect to $\omega$ and $k$ of the individual compound layer matrices and then using the chain rule.

In the absence of an atmosphere, $\left[p_{P_{\mathrm{a}}} / v_{0}\right]$ is zero. For a nongravitating isothermal ocean terminated at sea level by a free surface, $A_{21}$ and $A_{22}$ are given by

$$
A_{21}=-i c \rho_{0} \sin P_{0} / r_{\alpha} \quad A_{22}=\cos P_{0}
$$

where

$$
P_{0}=k r_{\alpha} d_{0} \quad r_{\alpha}=\left(c^{2} / \alpha^{2}-1\right)^{1 / 2}
$$

Thus equation (38) reduces to

$$
F_{\circ}=[N K-L M]+i\left(c^{2} \rho_{0} / r_{\alpha}\right) \tan P_{0}[G N-L H]
$$

which is the period equation for oceanic Rayleigh waves [Dorman, 1962; Harkrider and Anderson, 1962]. As a test of the program, we calculated dispersion values for a constant-velocity ocean model. These values were identical to roots obtained using an earlier program based on (50).

The models used in this paper consist of a standard ARDC atmosphere overlying four earth models. The layer parameters for the oceanic, shield, and tectonic models are those given by Harkrider and Anderson [1966] and Anderson and Harkrider [1968]. The Gutenberg continental model is tabulated by Ben-Menahem and Harkrider [1964]. The atmosphere is terminated by a free surface at an altitude of $310 \mathrm{~km}$.

The period equation, (38), determines the dispersion relation for all surface waves in the coupled system. The roots correspond not only to Rayleigh waves in the earth but also to acoustic-gravity waves in the atmosphere-ocean system. 
The Rayleigh waves are obtained by choosing the initial or trial wave number $k$ so that the nearest root is a Rayleigh wave mode. The fundamental and first higher-mode Rayleigh waves were calculated for the four models at periods ranging from 60 to $2.5 \mathrm{sec}$. Inclusion of the atmosphere changed the previously calculated free-surface values of phase velocity, group velocity, and medium response by less than $0.01 \%$.

The free-surface boundary condition at the top of the atmosphere was chosen in order to obtain real roots for the Rayleigh waves, since the alternate boundary condition of terminating the top of the atmosphere by an isothermal half-space causes the Rayleigh roots to be complex (at the periods of interest here), owing to the radiation of energy out of the atmospheric wave guide. An estimate of the imaginary part of $k$ has been obtained from a simple model. For a fluid half-space overlying a solid half-space with physical constants appropriate to the atmosphere and solid earth, $\operatorname{Im}(k)$ is less than $3 \times 10^{-5} \mathrm{~km}^{-1}$ [Cagniard, 1962, p. 224]. This estimate was made using the density at sea level and assuming a constant density throughout the atmosphere. Since $\operatorname{Im}(k)$ is proportional to the fluid-solid density ratio, we would expect this value to be a generous upper limit to a half-space termination at altitude with exponentially decreasing density. In any case, this value is an order of magnitude less than the attenuation coefficient due to anelasticity, and hence can be neglected.

The second program is a modification of the acoustic-gravity wave program used to calculate the atmosphere eigenfunctions $\left[p_{P_{m}}(z) / v_{0}\right]$ and $\left[v_{m}(z) / v_{0}\right]$ at specified altitudes. Input values of phase velocity $c$ and $\left[p_{P_{0}} / v_{0}\right]$ computed from the first program for a given period are continued by the matrix operation (24) to prescribed source altitudes. The source height excitation function $\left[p_{0}(D) / v_{0}\right]$ is then formed using (45).

Extending the layer matrix multiplication to the top of the atmosphere, we can calculate $\left[p_{P_{0}} / v_{0}\right]$ by the alternate expression 44 , where the first program uses the equivalent relation for the solid earth, (43), or

$$
\left[\frac{p_{P_{0}}}{v_{0}}\right]=-\frac{1}{c} \frac{[N K-L M]}{[G N-L H]}=-\frac{1}{c} \frac{\Re_{11}}{\Re_{12}}
$$

Since we can calculate $\left[p_{P_{0}} / v_{0}\right]$ in the eigenfunction program using the input $c$ and $T$, the input of $\left[p_{P_{0}} / v_{0}\right]$ is unnecessary. We include it as an input value only for computational convenience and for a check on the numerical results from the two programs. This was advisable because the second and third programs were run on an IBM 360/50, which has a smaller word length and hence fewer significant figures than the CDC 1604.

As was mentioned earlier, the effect of the atmospheric wave guide on the dispersion of Rayleigh waves is very small. Therefore a reasonable estimate of $\left[p_{s}(D) / v_{0}\right]$ can be obtained by using as input to an eigenfunction program the values of the Rayleigh roots at the free surface calculated from a solid-earth program. This fact can save a lot of programming effort for others interested in this problem.

The dispersion, solid-earth response, and source-height excitation values are used in a third program to synthesize vertical displacement seismograms for 
given input values of source yield, altitude, and distance to the observer. The program scales the source parameters and evaluates (47) using the Aki synthesis algorithm [Harkrider, 1964a].

The scaling rules are the same as those given by Harkrider [1964a] except that the scaled overpressure $p_{a s}$ should include as a correction the ratio of the ambient pressure at the actual source height and the standard source height [Pierce, 1965].

The values of the anelasticity absorption coefficient $\hat{\gamma}(\omega)$ used in the spectral decay factor exp $[-\hat{\gamma}(\omega) r]$ were obtained from a linear extrapolation of the longperiod observations of Ben-Menahem [1965]. At a period of 20 sec, the extrapolated value of $\hat{\gamma}(\omega)$ is $3.3 \times 10^{-4} \mathrm{~km}^{-1}$, which is in good agreement with the average oceanic value of $3 \times 10^{-4} \mathrm{~km}^{-1}$ observed by Gutenberg and Richter [1956].

The instrumental response included in the theoretical seismograms is that of a Geotech 20-30 seismometer system.

\section{NUMERICAL RESULTS}

Theoretical seismograms calculated at a distance of $10,000 \mathrm{~km}$ for a $1 \mathrm{kT}$ explosion are shown in Figures 2 and 3. The source altitudes in Figures 2 and 3 are 0.3 and $4.88 \mathrm{~km}$, respectively. A comparison of the fundamental oceanic Rayleigh wave ${ }_{1} O_{1}$ and first higher mode ${ }_{2} O_{1}$ at $0.3 \mathrm{~km}$ and $4.88 \mathrm{~km}$ indicate an enhancement of the long-period arrivals relative to the short periods at the higher source altitude. This is also true for the first higher shield mode ${ }_{2} S_{1}$. The effect of altitude at these heights on the fundamental shield mode ${ }_{1} S_{1}$ appears to be a reduction in amplitude of the intermediate frequencies in the middle of the wave train relative to the early-arriving long periods and late-arriving short periods. These particular altitudes were chosen only for illustrative examples of synthetic records and have no special significance.

The peak-to-trough vertical amplitudes of the fundamental mode at a period of $20 \mathrm{sec}$ is plotted as a function of altitude for the four models in Figure 4. The vertical displacements are calculated at the ocean bottom for the ${ }_{1} O_{1}$ mode and at sea level for the fundamental shield mode ${ }_{1} S_{1}$, the fundamental tectonic mode ${ }_{1} T_{1}$, and the Gutenberg continental mode, ${ }_{1} G_{1}$. For source altitudes between 0.3 and $3.66 \mathrm{~km}$, the oceanic amplitudes are 3 to 5 times larger than those

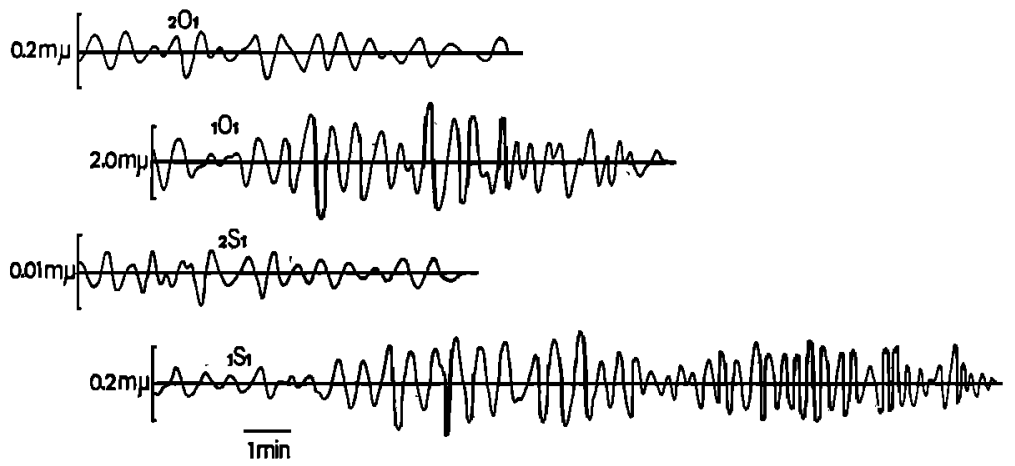

Fig. 2. Vertical displacements for a $1-\mathrm{kT}$ explosion at an altitude of $0.3 \mathrm{~km}$. 


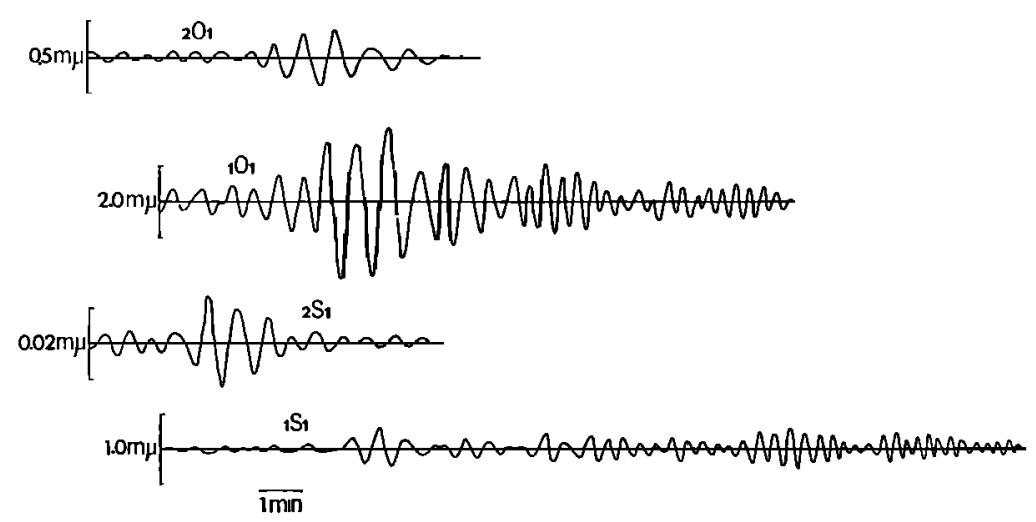

Fig. 3. Vertical displacements for a 1-kT explosion at an altitude of $4.88 \mathrm{~km}$.

calculated on land. Vertical displacements were used, since their depth profile at 20 sec was almost constant from the surface down into the upper crust. Thus these amplitudes do not reflect an amplification due to soft sedimentary layers in the upper part of the crust.

Observationally and theoretically, McGarr [1969] has found that, for Rayleigh waves crossing a continental margin, the ocean bottom amplitudes are 2 to 3 times smaller than amplitudes on land. Thus measurements observed on land for an explosion over an oceanic crust at this distance should be 6 to 15 times larger than those for an explosion over a continental crust observed at the same distance at a station on land. The models used in this paper are not identical to those used in the theoretical calculations of $M c G a r r$ [1969], but he has shown that an approximation to at least the direction of amplification can be obtained by assuming that the total energy in the fundamental mode is conserved on crossing the continental margin. Using this approximation, we have found that our models also show an amplification consistent in direction with his.

As has been stated, the same anelastic attenuation values were used for all models, and the values adopted are those appropriate for an oceanic path. At the assumed distance and periods, the observed smaller coefficients for land paths will increase the theoretical land-path amplitudes by as much as a factor of 12 [Gutenberg and Richter, 1956; Tryggvason, 1965]. Thus the preceding statements pertain only to observed data for which possible differences in attenuation along the propagation paths have been corrected.

In Figure 5, the ratio of the maximum amplitude for the first higher mode to the amplitude of the fundamental mode at 20-sec period is shown as a function of source height. All models have a minimum at a source height of from 1 to $2 \mathrm{~km}$. The cause of the difference between the oceanic and land models for sources above $3.66 \mathrm{~km}$ has not yet been determined, but the increase in relative excitation for the first higher mode over the fundamental with increasing altitude might prove to be a diagnostic aid for high-altitude explosions at sea.

An estimate of whether the effects in Figure 4 are due to coupling beneath the source or to dispersion along the path can be obtained by an approximate 
elimination of the amplitude dispersion factor. This factor, $U[d U / d T]^{-1 / 2}$, is given by the stationary phase approximation to (47). For our models at $20 \mathrm{sec}$ this factor, determined graphically, is approximately

$$
\begin{aligned}
& U[d U / d T]_{1 O_{1}{ }^{-1 / 2}} \approx 10.6 \\
& U[d U / d T]_{1 T 1}{ }^{-1 / 2} \approx 29.3 \\
& U[d U / d T]_{1 s 1}{ }^{-1 / 2} \approx 13.8 \\
& U[d U / d T]_{1 G 1}{ }^{-1 / 2} \approx 17.0
\end{aligned}
$$

Keeping in mind that $20 \mathrm{sec}$ is dangerously near the group velocity minimum for ${ }_{1} T_{1}$ and ${ }_{1} G_{1}$ (where the stationary phase approximation breaks down), we see that eliminating the dispersion effects by dividing out these factors accentuates the difference between the ocean and land models. Thus these curves seem to

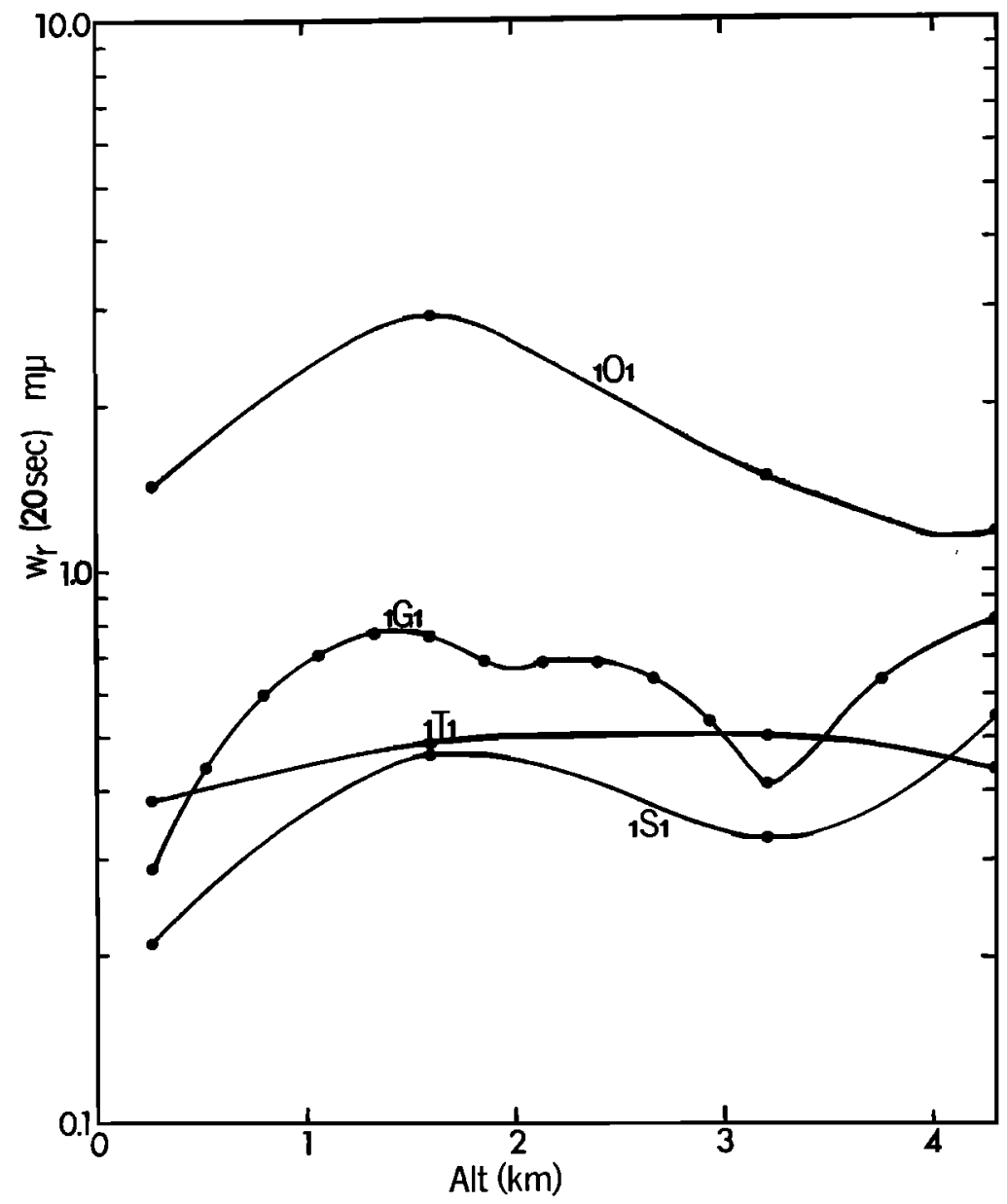

Fig. 4. Maximum vertical displacement at 20-sec period as a function of source altitude. 


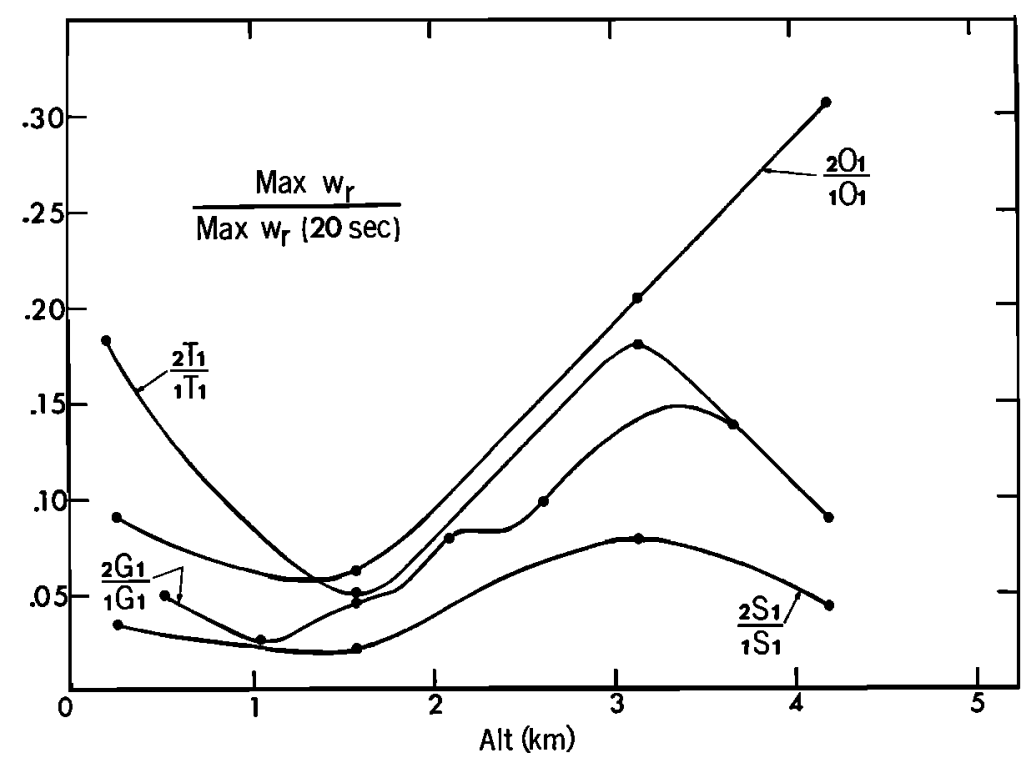

Fig. 5. Ratio of maximum first higher-mode displacement to the 20-sec fundamental mode displacement as a function of source altitude.

indicate that the larger amplitudes for the ocean model are due to better coupling between the atmosphere, ocean, and oceanic crust-upper mantle structure than between the atmosphere-continental structures.

\section{CONCLUSIONS}

We have compared the generation of Rayleigh waves by atmospheric explosions over oceanic structures and over three types of continental earth structures. We conclude that on the basis of the admittedly simplified models adopted here:

1. More efficient coupling of energy from the atmosphere results in approximately 3 to 5 times greater amplitude of vertical-component Rayleigh waves for the oceanic structure than for any of the three types of continental structure, at 20 sec period.

2. On the basis of McGarr's results, we conclude that the effect of continental margins should be to increase the amplitude of Rayleigh waves on the continent with respect to the ocean bottom.

3. Thus, an explosion over water that is observed (in the form of Rayleigh waves) on land should exhibit amplitudes an order of magnitude greater than the equivalent amplitudes from an explosion of the same burst height and yield over any of our three assumed continental structures.

4. However, the effect of anelastic absorption of Rayleigh waves is in the opposite direction: regardless of the earth structure immediately beneath the explosion, the fact that $Q$ is lower for oceanic crust-upper mantle structures than for any of our continental structures implies that observed Rayleigh-wave 
amplitudes at sufficiently large teleseismic distances should be greater for continental paths than for oceanic paths.

Acknowledgments. This research was supported at Brown University by the Advanced Research Projects Agency and was monitored by the Air Force Office of Scientific Research under contract AF-F44620-68-C-0082.

This research was partly supported at Geotech by the Advanced Research Projects Agency, Nuclear Test Detection Office, under Project VELA-Uniform and was under technical direction of the Air Force Technical Applications Center under contract F33657-68-C0945.

\section{REFERENCES}

Anderson, D. L., and D. G. Harkrider, Universal dispersion tables, 2, Variational parameters for amplitudes, phase velocity, and group velocity for first four Love modes for an oceanic and a continental earth model, Bull. Seismol. Soc. Amer., 58, 1407-1499, 1968.

Bäth, M., Seismic records of explosions-especially nuclear explosions, 3, Försvarets Forskningsanstalt Avdelning 4 rapports A 4270-4721, Uppsala, 116 pp., 1962.

Ben-Menahem, A., Observed attenuation and $Q$ values of seismic surface waves in the upper mantle, J. Geophys. Res., 70, 4641-4652, 1965.

Ben-Menahem, A., and D. G. Harkrider, Radiation patterns of seismic surface waves from buried dipolar point sources in a flat stratified earth, J. Geophys. Res., 69, 2605-2620, 1954.

Cagniard, L., Reflection and Refraction of Progressive Seismic Waves, translated and revised by E. A. Flinn and C. H. Dix, McGraw-Hill, New York, 1962.

Crampin, S., Higher-mode seismic surface waves from atmospheric nuclear explosions over Novaya Zemlya, J. Geophys. Res., 71, 2951-2958, 1966.

Dorman, J., Period equation for waves of Rayleigh type on a layered, liquid-solid half space, Bull. Seismol. Soc. Amer., 52, 389-397, 1962.

Dunkin, J. W., Computation of modal solutions in layered, elastic media at high frequencies, Bull. Seismol. Soc. Amer., 65, 335-358, 1965.

Erdelyi, A., W. Magnus, F. Oberhettinger, and F. Tricomi, Tables of Integral Transforms, vol. 2, p. 9, McGraw-Hill, New York, 1954.

Gilbert, F., and G. E. Backus, Propagator matrices in elastic wave and vibration problems, Geophysics, 31, 326-332, 1966.

Glasstone, S., The Effects of Nuclear Weapons, U.S. Government Printing Office, Washington, D. C., 1962.

Gutenberg, B., and C. F. Richter, Earthquake magnitude, intensity, energy, and acceleration, Bull. Seismol. Soc. Amer., 46, 105-145, 1956.

Harkrider, D. G., Theoretical and observed acoustic-gravity waves from explosive sources in the atmosphere, J. Geophys. Res., 69, 5295-5321, $1964 a$.

Harkrider, D. G., Surface waves in multilayered elastic media, 1, Rayleigh and Love waves from buried sources in a multilayered elastic half-space, Bull. Seismol. Soc. Amer., 54, $627-679,1964 b$.

Harkrider, D. G., Surface waves in multilayered elastic media, 2, Higher mode spectra and spectral ratios from point sources in plane layered earth models, submitted to Bull. Seismol. Soc. Amer. 1970.

Harkrider, D. G., and D. L. Anderson, Computation of surface wave dispersion for multilayered anisotropic media, Bull. Seismol. Soc. Amer., 52, 321-332, 1962.

Harkrider, D. G., and D. L. Anderson, Surface wave energy from point sources in plane layered earth models, J. Geophys. Res., 71, 2967-2930, 1966.

Harkrider, D., and F. Press, The Krakatoa air-sea waves: An example of pulse propagation in coupled systems, Geophys. J., 13, 149-159, 1967.

McGarr, A., Amplitude variations of Rayleigh waves: Propagation across a continental margin, Bull. Seismol. Soc. Amer., 59, 1281-1306, 1969.

Nickel, G., and W. Whitaker, Distant seismic waves from a high-altitude source, Air Force 
Weapons Lab. Tech. Rep. AFWL-TR-67-125, 1, Kirtland Air Force Base, New Mexico, 42 pp., 1968.

Pierce, A. D., Comments on paper by David G. Harkrider, "Theoretical and observed acoustic-gravity waves from explosive sources in the atmosphere,' J. Geophys. Res., 70, 2463-2464, 1965.

Press, F., and D. Harkrider, Propagation of acoustic-gravity waves in the atmosphere, $J$. Geophys. Res., 67, 3889-3908, 1962.

Thrower, E. N., The computation of dispersion curves on layered media, J. Sound Vib., 2, 14-30, 1965.

Tryggvason, E., Dissipation of Rayleigh wave energy, J. Geophys. Res., 70, 1449-1455, 1965.

Toksöz, M. N., and A. Ben-Menahem, Excitation of seismic surface waves by atmospheric nuclear explosions, J. Geophys. Res., 69, 1639-1648, 1964.

(Typescript received December 18, 1968; revised July 23, 1969.) 